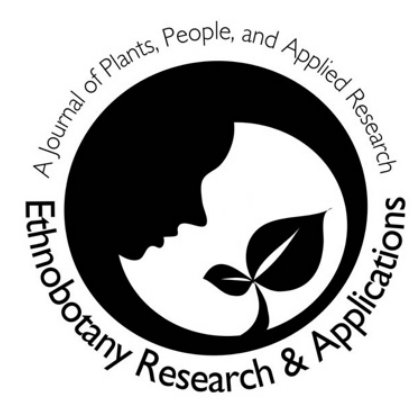

\title{
Ethnobotanical study of medicinal plants used by Miao people in Jijiezi, Yunnan, China
}

\author{
Yong Ma, Dongyang Liu, Hong Cheng, Rainer W. \\ Bussmann, Huajie He, Zhiyong Guo, Bo Liu
}

\section{Research}

\begin{abstract}
Background: The Miao ethnic group is an indigenous group in China with a long history of traditional medicine utilization and owns rich traditional knowledge related to biodiversity utilization and conservation. In Jijiezi village, Yunnan, the Miao ethnic group is the main ethnic group and retains a rich traditional culture. The area is rich in plant resources and the custom of using Miao medicine is still retained. It is important to evaluate the inheritance of the knowledge of Miao medicine in the region and the development prospects of Miao medicine culture.
\end{abstract}

Methods: The data was collected in different seasons in 2014, with a total of 200 informants interviewed using free listing and semi-structured interviews.

Results: This study recorded the ethnobotanical importance of Miao medicine in Jijiezi, Yunnan, China. 42 species from 26 families and 37 genera were identified. For each species scientific and Miao names, distribution, and use knowledge were recorded and analyzed which can help the assessment the current status of Miao traditional medicine. The results show that the traditional knowledge of Miao medicine is very rich and has a good therapeutic effect on a variety of diseases, and that there is a homology of medicine and food. However, the traditional knowledge of Miao medicine is more and more getting lost and needs urgent protection.

Conclusions: It is important to protect the traditional Miao medicine knowledge and promote sustainable development of Miao medicine culture. We propose to protect and develop local Miao medicine knowledge, and suggest (1) documentation of species used in this practice may provide basic information for conservation, and further use, and will help to preserve local traditional knowledge, (2) training of practitioners in Miao medicine to maintain Miao medicine culture, (3) encourage local governments to adopt relevant policies to protect the culture of Miao medicine, and (4) bring Miao medicine into the market and promoting the development of Miao medicine.

Keywords: Miao nationality, traditional knowledge, Medicinal plants, Yunnan, Biodiversity

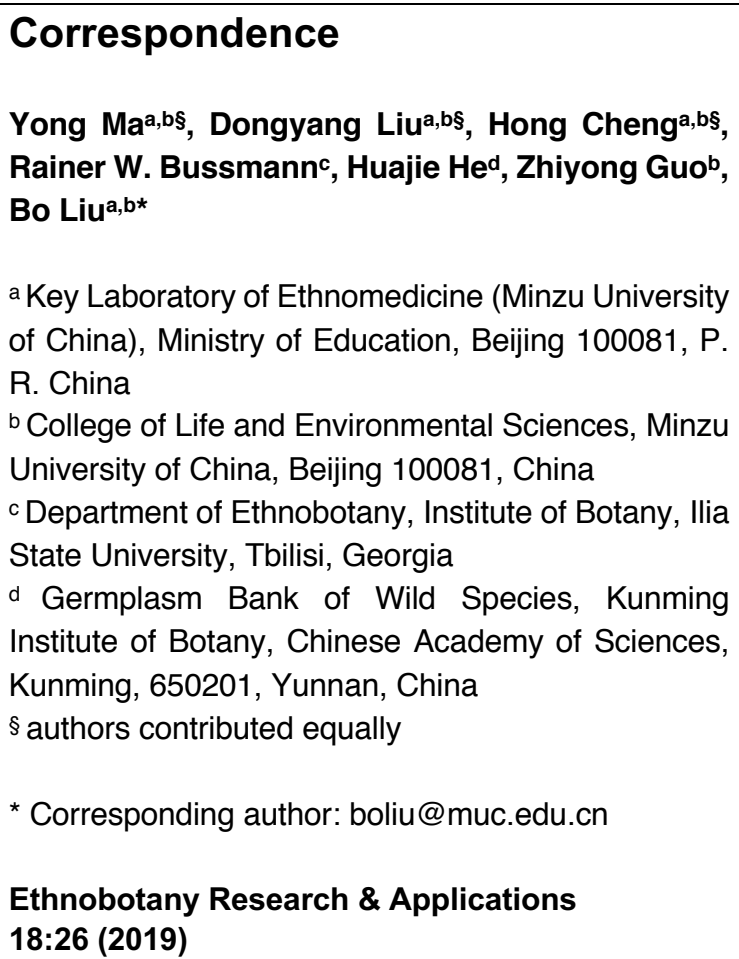

Yong Maa,b§, Dongyang Liua,bs, Hong Chenga,bs, Rainer W. Bussmannc, Huajie Hed, Zhiyong Guob, Bo Liu ${ }^{a, b *}$

a Key Laboratory of Ethnomedicine (Minzu University of China), Ministry of Education, Beijing 100081, P. R. China

${ }^{b}$ College of Life and Environmental Sciences, Minzu University of China, Beijing 100081, China

c Department of Ethnobotany, Institute of Botany, Ilia State University, Tbilisi, Georgia

Institute of Botany, Chinese Academy of Sciences, Kunming, 650201, Yunnan, China 18:26 (2019) 


\section{摘要 (Abstract in Chinese):}

背景: 苗族是中国的一个世居民族, 有很悠久的传统 药利用历史和丰富的传统知识。云南的鸡街子村中主 体民族为苗族, 保留了很丰富的传统文化。该地区有 丰富的植物资源, 村民主要以种植植物和养殖业为生 , 发展较为落后。因此, 该地区依然保留了使用苗药 的风俗习惯。评价该地区对苗药知识的传承程度, 以 及苗药文化的发展前景是十分有意义的。

方法: 在2014年的不同季节, 采用自由列举法和半 结构式访谈, 对200个当地苗族进行采访来获得数据

结果: 本研究记录了在中国云南鸡街子村的苗药在民 族植物学方面的重要性。鉴别了26科 37 属的42种植

物, 记录和分析了每种物种的学名和苗名, 分布和使 用的经验, 这些能够帮助我们评估苗族传统药物的现 状。结果表明, 苗药传统知识十分丰富, 对于当地疾 病有较好的治疗效果, 且存在着药食同源的现象, 但 是目前苗药传统知识流失严重, 亟需对此进行保护。 结论: 保护这个地区的传统苗药知识以及实现其可持 续是非常重要的, 因此提出了保护和发展当地苗药知 识的建议: (1) 通过将这些植物物种信息编篡成册 能够为利用这些植物提供基础资料, 同时也能保护当 地传统知识; (2) 寻找苗药的继承人, 将苗药文化 传承下去;（3）当地政府应采取相关政策来保护苗 药文化; (4) 将苗药引入市场, 通过经济促进苗药 发展。以此为苗族非物质文化遗产的传承提供帮助。 关键词: 苗族; 传统知识; 药用植物; 云南; 生物多 样性

\section{Background}

Even with increasing industrialization, traditional medicine is attracting more and more attention in the world (Jäger et al. 2006, Tsai et al. 2008). At the same time, the knowledge of traditional medicine in many regions of Chinais widely disappearing because of the impact of Western medical culture and the Han medical culture in China (Janes 1999). The Miao ethnic group is one of the most ancient ethnic groups in China and regarded as the first ethnic group in China Miao rice (Pan, 1996). A long history of farming and settlement have made the Miao famous for thier discoveries and domestication of medicinal plants. Even as migrants the Miao are well known to keep their home medicinal gardens traditions (Wu, 2004). Many medicinal plant resources are widely used by Miao people (Anderson et al. 2005, Long \& Rong 2004, Shen et al. 2010, Zhang et al. 2015). The Miao have inhabited their homeland of Yunnan and Guizhou provinces for thousands of years. Over time the population has accumulated rich traditional medical knowledge. Miao people from Jijiezi village (Yunnan Province) belongs to the Weining branch of the ethnic group, calling themselves the "base branch". The old ways of village folk customs, slash-and-burn agriculture, hemp weaving and batik embroidery still exist. Because Miao people in Jijiezi have been isolated from other parts of the ethnic group for a long time, they have developed different uses of many medicinal plants. For example, Miao people in Guizhou province use "herbal medicine bath" frequently, but Miao people in Jijiezi do not have this tradition based on our observations and interviews. Among Chinese ethnic groups, Miao has more branches than any other groups. The Miao people from Jijiezi village (Yunnan Province) belongs to the Weining branch, they call themselves "basic branch". This basic branch is likely to have been suppressed after failure in fighting against the Qing Dynasty in the east and west of Guizhou province, and then moved to the Jijiezi village in Yunnan Province. The old traditions such as village folk customs, swidden agriculture, natural fiber woven products and batik embroidery still exist. Since the Miao people in Jijiezi have been isolated from other branches for a long time, the use of many medicinal plants and pharmaceutical operating methods are divergent now. Many traditional medicinal herbs have limited distributions in Guizhou, and when they moved to Yunnan, fewer herbs available, so they lost some old medicinal species and try to explore new plants in Yunnan local areas. Another example, the Miao people in Guizhou province use "herbal medicine bath" frequently, but the Miao people in Jijiezi do not have this tradition.

Miao medicine is regarded as simple, convenient, effective and inexpensive and has had far-reaching influence in local medicinal culture. Its unique curative effects (Cheng et al. 2013, Xiong et al. 2010) are based on plant material from the natural environment, and it is closely linked to local people's lifestyle, which can make a significant contribution to health. According to the relevant research (Zheng 2006) documentation of Yunnan Miao medicine is greatly lacking. There is an urgent needed to protect this valuable ethnic medicine knowledge for the 
world.

The aim of this study was to: (1) record traditional Miao medicine used by local people in Jijiezi, (2) discover the cultural heritage passed down from generations to generations by local Miao people, (3) document associated ethnobotanical knowledge, and (4) give some suggestions about protection of local valuable medicinal system.

\section{Materials and methods}

Study area

Jijiezi village is located in Yunnan Province, Southwestern China. It has over 3000 residents from three ethnic groups: Miao, Yi and Han, with Miao people accounting for more than $95 \%$ of the population. This area has a mean elevation of 2160 $\mathrm{m}$, an annual average temperature of $19.5^{\circ} \mathrm{C}$, and an annual average rainfall of $650 \mathrm{~mm}$ (Fig. 1).

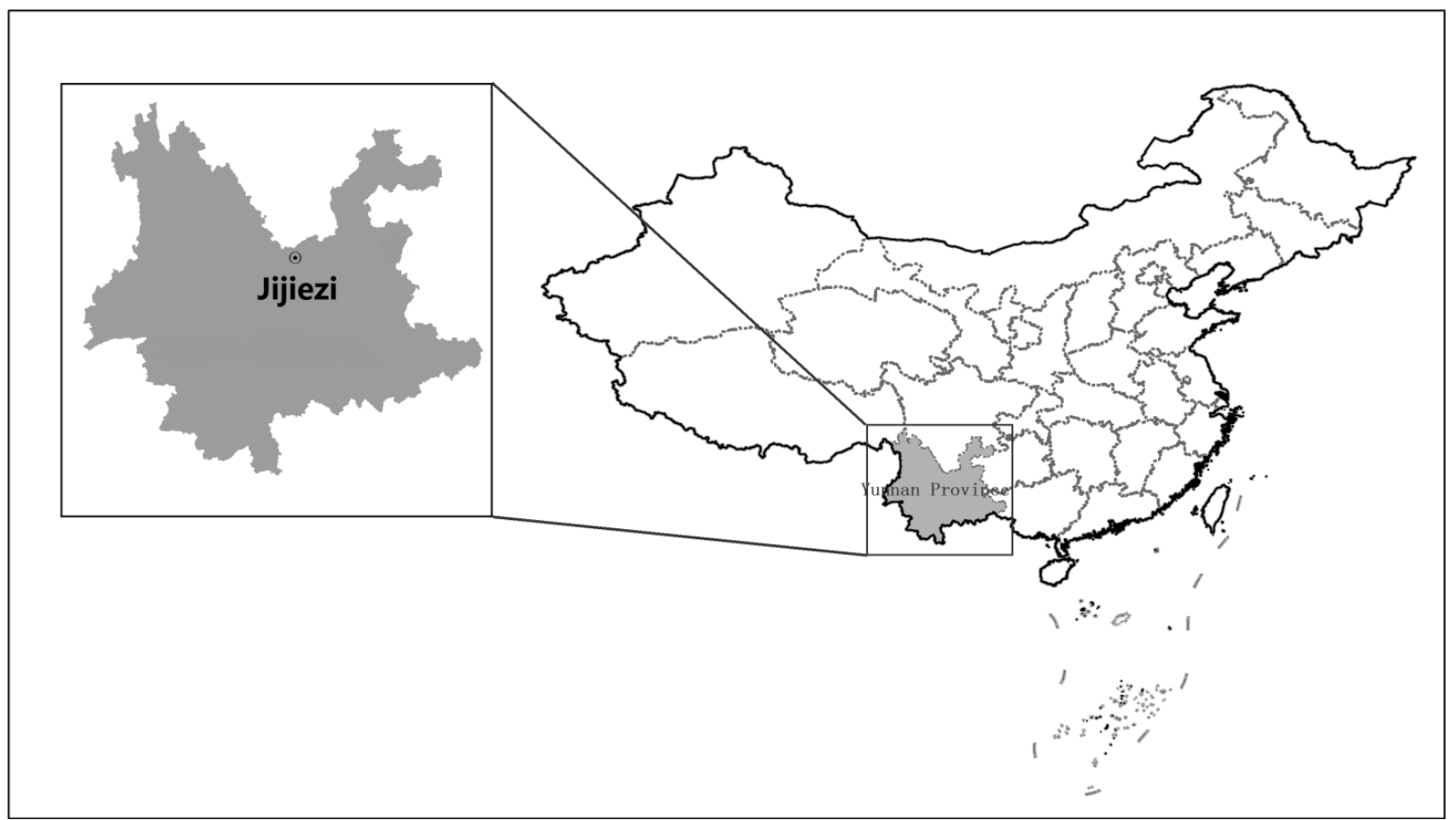

Fig. 1. Location of Jijiezi village, Yunnan, China

To conduct the study we first obtained permission from Yunnan government, and collected general geological and historical information on distributions of Miao people, and their traditions. On the basis of this information, we selected Jijiezi village as a representative village for our research. Jijiezi village is famous for its rich plant resources, and its forest coverage reached $46.64 \%$. Most people still keep the traditional Miao customs.

\section{Research methods}

A total of 200 local Miao people including 100 men and 100 women were interviewed after obtaining prior informed consent. The demographics of the participants are given in Table 1. Local herb markets were also visited in different seasons in 2014 (Fig. 2). Key informants included local guides, drivers and five local Miao practitioners.
From the interviews, we obtained information on vernacular names and uses of medicinal plants, collection localities, production data, common diseases treated, medicinal plant related habitats and harvesting time (Table 1 \& Table 2). Voucher specimens have been collected and stored in the herbarium of Minzu University of China.

\section{Results and discussion}

\section{The most popular medicinal plants}

Local medicinal plant species in Jijiezi are very diverse. In our study, we found 42 species frequently used as Miao medicine by local practitioners (Table 2). The most frequently used plants by Miao people include Houttuynia cordata Thunb., Lonicera japonica Thunb., Platycodon grandiflorus (Jacq.) A. DC., Arisaema heterophyllum Blume, and Sophora flavescens Alt. 

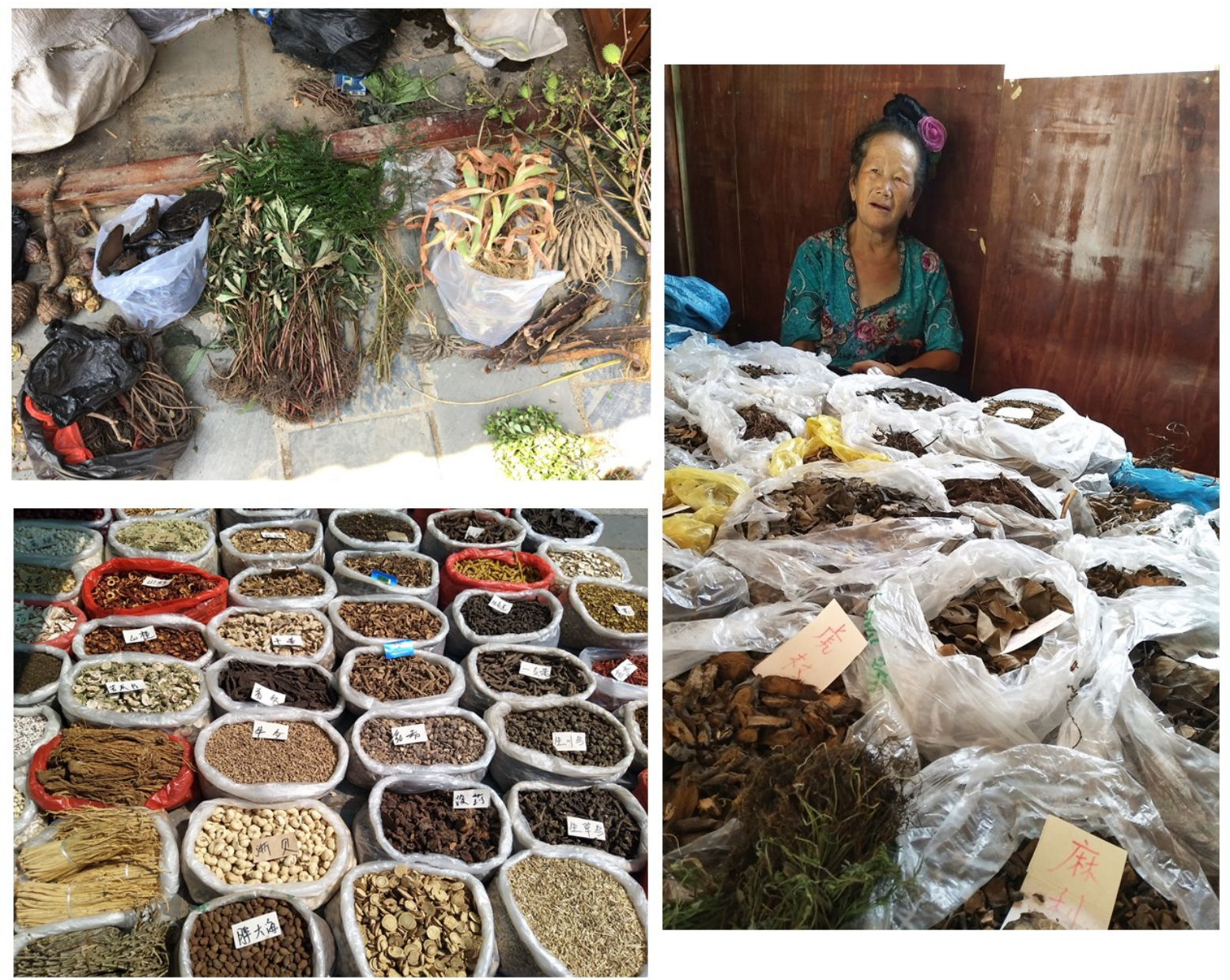

Fig. 2. Miao medicine market in Jijiezi village

Phyllanthus emblica L., Chloranthus japonicus Siebold, Paris polyphylla Sm., Chloranthus japonicus Siebold were mentioned by almost each interviewee. Allium sativum L., Allium tuberosum Rottler ex Spreng and Phyllanthus emblica L. were used both as medicinal and edible plants, which indicates that the homology of medicine and food is very popular. Some local Miao medicines like Paris polyphylla Sm., are one of the main components in the drug Yunnan Baiyao (Chinese: 云南白药) for traumatic injuries. Because of its good therapeutic effects and large demand, wild Paris polyphylla Sm. suffers from over-exploitation. and is in danger of extinction.

An overview on plant parts used is given in (Fig. 3).

This result are similar to other investigations, such as a study of the wild edible plants used by Tibetans in Shangri-la region, Yunnan, China (Yan et al. 2012).

Table 1. Demographic features of the participants $\mathrm{n}=200$

\begin{tabular}{|c|c|c|}
\hline & Number & Percentage \\
\hline Gender & 40 & $20 \%$ \\
\hline $\begin{array}{c}\text { Adolescents } \\
\text { (aged under 18 } \\
\text { years) }\end{array}$ & 40 & $20 \%$ \\
\hline $\begin{array}{c}\text { Adult men } \\
\text { (aged 18-45 } \\
\text { years) }\end{array}$ & 40 & $20 \%$ \\
\hline $\begin{array}{c}\text { Adult women } \\
\text { (aged 18-45 } \\
\text { years) }\end{array}$ & 40 & $20 \%$ \\
\hline $\begin{array}{c}\text { Elderly men } \\
\text { (aged over } 45 \\
\text { years) }\end{array}$ & 40 & $82 \%$ \\
\hline $\begin{array}{c}\text { Elderly women } \\
\text { (aged over } 45 \\
\text { years) }\end{array}$ & 164 & $18 \%$ \\
\hline Origin & 36 & \\
\hline $\begin{array}{c}\text { Local Miao } \\
\text { farmers }\end{array}$ & & \\
\hline $\begin{array}{c}\text { Local other } \\
\text { farmers }\end{array}$ & & \\
\hline
\end{tabular}




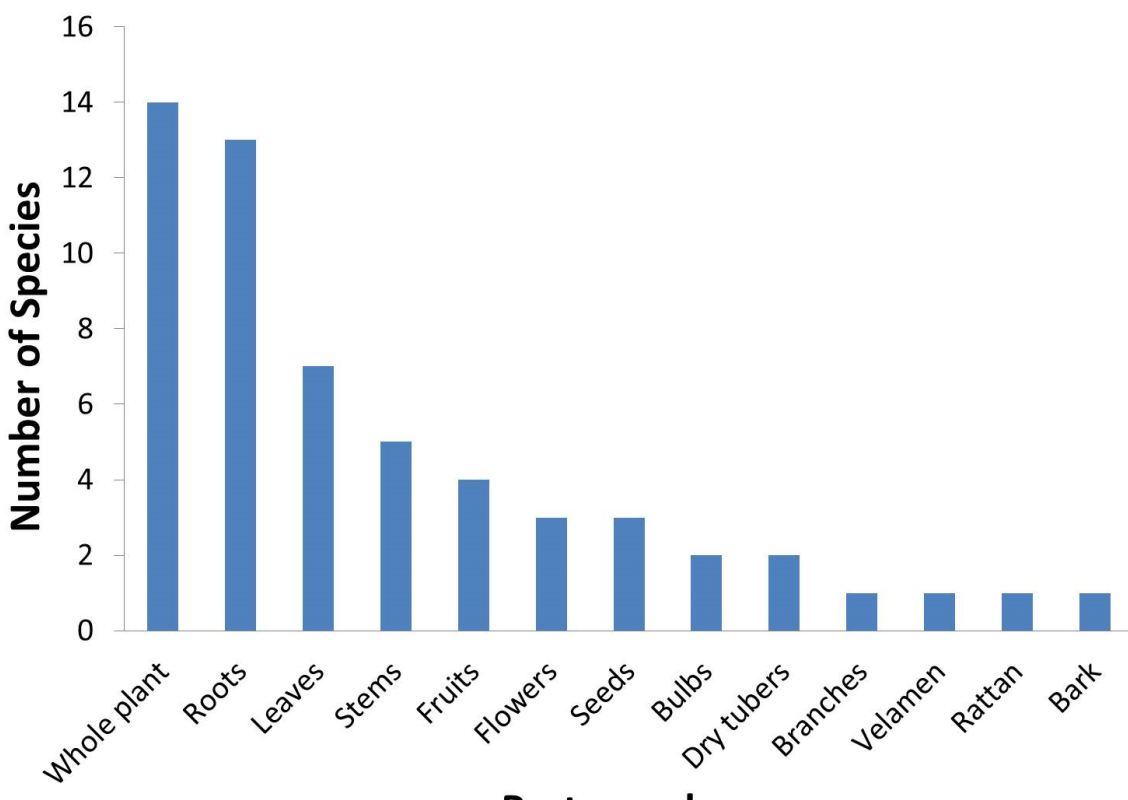

Parts used

Fig. 3. Use frequency of Miao medicinal herb parts.

Table 2. Miao medicinal plants used in Jijiezi village

\begin{tabular}{|c|c|c|c|c|c|c|}
\hline $\begin{array}{l}\text { Family and } \\
\text { Scientific name }\end{array}$ & $\begin{array}{l}\text { Miao and } \\
\text { Chinese } \\
\text { name }\end{array}$ & $\begin{array}{l}\text { Parts } \\
\text { used }\end{array}$ & Habitat & $\begin{array}{l}\text { Main } \\
\text { chemical } \\
\text { compounds }\end{array}$ & $\begin{array}{l}\text { Traditional use and } \\
\text { applications }\end{array}$ & $\begin{array}{l}\text { Voucher } \\
\text { specimen }\end{array}$ \\
\hline \multicolumn{7}{|l|}{ Amaryllidaceae } \\
\hline Allium sativum $\mathrm{L}$. & $\begin{array}{l}\text { Miao: da } \\
\text { suan } \\
\text { 蒜 } \\
\text { suan }\end{array}$ & Bulbs & Farmland & $\begin{array}{l}\text { Allicin } \\
\text { (Baghalian et } \\
\text { al. 2005; } \\
\text { Blania and } \\
\text { Spangenberg } \\
\text { 1991) }\end{array}$ & $\begin{array}{l}\text { Resistance of cold, } \\
\text { cough. Harvest time: } \\
\text { throughout the year }\end{array}$ & $\begin{array}{l}\text { Liu } \\
\text { Dongyang, } \\
\text { Guo Zhiyong } \\
\text { et al. } 1\end{array}$ \\
\hline $\begin{array}{l}\text { Allium tuberosum } \\
\text { Rottler ex Spreng. }\end{array}$ & $\begin{array}{l}\text { Miao: jiu cai } \\
\text { 非 } \\
\text { jiu }\end{array}$ & $\begin{array}{l}\text { Seeds, } \\
\text { leaves }\end{array}$ & Farmland & $\begin{array}{l}\text { Sulfides, } \\
\text { glycosides } \\
\text { (Wu and } \\
\text { Zhang 2005) }\end{array}$ & $\begin{array}{l}\text { Treat nocturnal } \\
\text { emission, enuresis, } \\
\text { frequent urination } \\
\text { (Hu et al. 2013). } \\
\text { Harvest time: } \\
\text { throughout the year }\end{array}$ & $\begin{array}{l}\text { Liu } \\
\text { Dongyang, } \\
\text { Guo Zhiyong } \\
\text { et al. } 2\end{array}$ \\
\hline $\begin{array}{l}\text { Lycoris aurea } \\
\text { (L'Hér.) Herb. }\end{array}$ & $\begin{array}{l}\text { 忽地笑 } \\
\text { hu di xiao }\end{array}$ & Bulbs & $\begin{array}{l}\text { Humid } \\
\text { areas }\end{array}$ & $\begin{array}{l}\text { Lycorine, } \\
\text { alkaloids } \\
\text { (Tong et al. } \\
\text { 2011) }\end{array}$ & $\begin{array}{l}\text { External use for itch, } \\
\text { rheumatism and joint } \\
\text { pain. Harvest time: } \\
\text { autumn }\end{array}$ & $\begin{array}{l}\text { Liu } \\
\text { Dongyang, } \\
\text { Guo Zhiyong } \\
\text { et al. } 9\end{array}$ \\
\hline \multicolumn{7}{|l|}{ Apiaceae } \\
\hline $\begin{array}{l}\text { Ligusticum sinense } \\
\text { Oliv. }\end{array}$ & $\begin{array}{l}\text { Miao: xiang } \\
\text { gao ben } \\
\text { 菜本gao } \\
\text { ben }\end{array}$ & $\begin{array}{l}\text { Roots, } \\
\text { stems }\end{array}$ & $\begin{array}{l}\text { Grows in } \\
\text { forests } \\
\text { and river } \\
\text { bank }\end{array}$ & & $\begin{array}{l}\text { Treat acariasis and } \\
\text { neurodermatitis }\end{array}$ & $\begin{array}{l}\text { Liu } \\
\text { Dongyang, } \\
\text { Guo Zhiyong } \\
\text { et al. } 4\end{array}$ \\
\hline \multicolumn{7}{|l|}{ Araceae } \\
\hline $\begin{array}{l}\text { Arisaema } \\
\text { heterophyllum } \\
\text { Blume. }\end{array}$ & $\begin{array}{l}\text { Miao: ma ti } \\
\text { jue ming } \\
\text { 决明 } \\
\text { jue ming }\end{array}$ & $\begin{array}{l}\text { Whole } \\
\text { plant }\end{array}$ & $\begin{array}{l}\text { Thickets } \\
\text { on slopes }\end{array}$ & $\begin{array}{l}\text { Seeds contain } \\
\text { anthracene } \\
\text { glycosides } \\
\text { substances } \\
\text { and xanthones } \\
\text { (Murshid et al. } \\
\text { 2007) }\end{array}$ & $\begin{array}{l}\text { Treat hypertension, } \\
\text { headaches and } \\
\text { constipation. } \\
\text { Harvest time: } \\
\text { autumn }\end{array}$ & $\begin{array}{l}\text { Liu } \\
\text { Dongyang, } \\
\text { Guo Zhiyong } \\
\text { et al. } 5\end{array}$ \\
\hline \multicolumn{7}{|l|}{ Asteraceae } \\
\hline Bidens pilosa L. & $\begin{array}{l}\text { Miao: zhan } \\
\text { ren cao鬼针 } \\
\text { 草gui zhen } \\
\text { cao }\end{array}$ & $\begin{array}{l}\text { Whole } \\
\text { plant }\end{array}$ & $\begin{array}{l}\text { Villages, } \\
\text { roadsides, } \\
\text { wasteland. }\end{array}$ & $\begin{array}{l}\text { Hyperoside, } \\
\text { gallic acid, } \\
\text { flavonoids }\end{array}$ & $\begin{array}{l}\text { Treat appendicitis, } \\
\text { gastroenteritis, } \\
\text { arthralgia, External } \\
\text { used treat boils. }\end{array}$ & $\begin{array}{l}\text { Liu } \\
\text { Dongyang, } \\
\text { Guo Zhiyong } \\
\text { et al. } 22\end{array}$ \\
\hline
\end{tabular}




\begin{tabular}{|c|c|c|c|c|c|c|}
\hline $\begin{array}{l}\text { Campanulaceae } \\
\text { Platycodon } \\
\text { grandiflorus (Jacq.) } \\
\text { A. DC. }\end{array}$ & $\begin{array}{l}\text { Miao: ling } \\
\text { dang hua桔 } \\
\text { 梗jie geng }\end{array}$ & Roots & $\begin{array}{l}\text { At around } \\
2000 \mathrm{~m} \text { in } \\
\text { mountains }\end{array}$ & & $\begin{array}{l}\text { Anticancer and } \\
\text { antidiabetics }\end{array}$ & $\begin{array}{l}\text { Liu } \\
\text { Dongyang, } \\
\text { Guo Zhiyong } \\
\text { et al. } 26\end{array}$ \\
\hline \multicolumn{7}{|l|}{ Capparaceae } \\
\hline $\begin{array}{l}\text { Capparis masaikai } \\
\text { Levl. }\end{array}$ & $\begin{array}{l}\text { Miao: tai ji } \\
\text { zi } \\
\text { 马槟榔ma } \\
\text { bing lang }\end{array}$ & Seeds & $\begin{array}{l}\text { Valleys or } \\
\text { hillsides }\end{array}$ & $\begin{array}{l}\text { Emodin, } \\
\text { chrysophanic } \\
\text { acid }\end{array}$ & $\begin{array}{l}\text { Nourishing lung and } \\
\text { throat and expedite } \\
\text { delivery. Harvest } \\
\text { time: winter }\end{array}$ & $\begin{array}{l}\text { Liu } \\
\text { Dongyang, } \\
\text { Guo Zhiyong } \\
\text { et al. } 7\end{array}$ \\
\hline \multicolumn{7}{|l|}{ Caricaceae } \\
\hline Carica papaya L. & $\begin{array}{l}\text { Miao: yang } \\
\text { qie zi番木瓜 } \\
\text { fan mu gua }\end{array}$ & $\begin{array}{l}\text { Fruits, } \\
\text { leaves }\end{array}$ & $\begin{array}{l}\text { Widely } \\
\text { cultivated } \\
\text { in the } \\
\text { south of } \\
\text { Yunnan } \\
\text { province }\end{array}$ & $\begin{array}{l}\text { Phenolic } \\
\text { compounds, } \\
\text { coumarin } \\
\text { (Canini et al. } \\
\text { 2007) }\end{array}$ & $\begin{array}{l}\text { Treat stomachache. } \\
\text { Harvest time: } \\
\text { throughout the year }\end{array}$ & $\begin{array}{l}\text { Liu } \\
\text { Dongyang, } \\
\text { Guo Zhiyong } \\
\text { et al. } 8\end{array}$ \\
\hline \multicolumn{7}{|l|}{ Chloranthaceae } \\
\hline $\begin{array}{l}\text { Sarcandra glabra } \\
\text { (Thunb.) Nakai }\end{array}$ & $\begin{array}{l}\text { Miao: zhu } \\
\text { jie cao草珊 } \\
\text { 瑚cao shan } \\
\text { hu }\end{array}$ & $\begin{array}{l}\text { Whole } \\
\text { plant }\end{array}$ & $\begin{array}{l}\text { Hillsides, } \\
\text { shade of } \\
\text { the valley }\end{array}$ & $\begin{array}{l}\text { Volatile oil, } \\
\text { tannin }\end{array}$ & $\begin{array}{l}\text { Treat influenza. In } \\
\text { recent years, it has } \\
\text { also been used to } \\
\text { treat pancreatic } \\
\text { cancer and stomach } \\
\text { cancer, which has } \\
\text { no side effects. }\end{array}$ & $\begin{array}{l}\text { Liu } \\
\text { Dongyang, } \\
\text { Guo Zhiyong } \\
\text { et al. } 3\end{array}$ \\
\hline \multicolumn{7}{|l|}{ Convolvulaceae } \\
\hline $\begin{array}{l}\text { Cuscuta chinensis } \\
\text { Lam. }\end{array}$ & $\begin{array}{l}\text { Miao: wu } \\
\text { niang teng } \\
\text { 菟丝子tu si } \\
\text { zi }\end{array}$ & seeds & $\begin{array}{l}\text { Mountain } \\
\text { slopes }\end{array}$ & $\begin{array}{l}\text { Resin } \\
\text { glucoside, } \\
\text { vitamins, } \\
\text { carotene }\end{array}$ & $\begin{array}{l}\text { Nourish liver and } \\
\text { kidney; enhance the } \\
\text { energy, anti- } \\
\text { diarrhea. }\end{array}$ & $\begin{array}{l}\text { Liu } \\
\text { Dongyang, } \\
\text { Guo Zhiyong } \\
\text { et al. } 11\end{array}$ \\
\hline \multicolumn{7}{|l|}{ Eucommiaceae } \\
\hline $\begin{array}{l}\text { Eucommia ulmoides } \\
\text { Oliver }\end{array}$ & $\begin{array}{l}\text { Miao: jiao } \\
\text { shu } \\
\text { 杜仲du } \\
\text { zhong }\end{array}$ & Bark & Valleys & & $\begin{array}{l}\text { Nourish liver and } \\
\text { kidney, strengthen } \\
\text { the bones and } \\
\text { muscles }\end{array}$ & $\begin{array}{l}\text { Liu } \\
\text { Dongyang, } \\
\text { Guo Zhiyong } \\
\text { et al. } 12\end{array}$ \\
\hline \multicolumn{7}{|l|}{ Euphorbiaceae } \\
\hline $\begin{array}{l}\text { Phyllanthus emblica } \\
\text { L. }\end{array}$ & $\begin{array}{l}\text { Miao: wang } \\
\text { guo余甘子 } \\
\text { yu gan zi }\end{array}$ & $\begin{array}{l}\text { Fruits, } \\
\text { roots, } \\
\text { leaves }\end{array}$ & $\begin{array}{l}\text { Tolerance } \\
\text { of drought } \\
\text { and } \\
\text { barren, } \\
\text { like light } \\
\text { and } \\
\text { warmth }\end{array}$ & $\begin{array}{l}\text { Gallic acid, } \\
\text { ellagic acid et } \\
\text { al. (Zheng et } \\
\text { al. 2013) }\end{array}$ & $\begin{array}{l}\text { Fruits relieve cough } \\
\text { and sore throat, } \\
\text { roots and leaves } \\
\text { treat fever and } \\
\text { eczema. Harvest } \\
\text { time: summer, } \\
\text { autumn }\end{array}$ & $\begin{array}{l}\text { Liu } \\
\text { Dongyang, } \\
\text { Guo Zhiyong } \\
\text { et al. } 13\end{array}$ \\
\hline \multicolumn{7}{|l|}{ Fabaceae } \\
\hline $\begin{array}{l}\text { Albizia julibrissin } \\
\text { Durazz. }\end{array}$ & $\begin{array}{l}\text { Miao: ma } \\
\text { ying hua } \\
\text { 合欢he } \\
\text { huan }\end{array}$ & $\begin{array}{l}\text { Velamen } \\
\text {, flowers }\end{array}$ & Thickets & $\begin{array}{l}\text { Velamen } \\
\text { contains } \\
\text { saponins and } \\
\text { tannins (Xu et } \\
\text { al. 2008) }\end{array}$ & $\begin{array}{l}\text { Treat neurasthenia, } \\
\text { insomnia and } \\
\text { forgetfulness. } \\
\text { Harvest time: } \\
\text { summer, autumn }\end{array}$ & $\begin{array}{l}\text { Liu } \\
\text { Dongyang, } \\
\text { Guo Zhiyong } \\
\text { et al. } 14\end{array}$ \\
\hline $\begin{array}{l}\text { Alysicarpus vaginalis } \\
\text { (L.) DC. }\end{array}$ & $\begin{array}{l}\text { Miao: xiao } \\
\text { dou } \\
\text { 链英豆lian } \\
\text { jia dou }\end{array}$ & $\begin{array}{l}\text { Whole } \\
\text { plant }\end{array}$ & $\begin{array}{l}\text { Open } \\
\text { slopes, dry } \\
\text { field edge, } \\
\text { roadside }\end{array}$ & & $\begin{array}{l}\text { Promote blood } \\
\text { circulation, treat } \\
\text { wounds, snake bite } \\
\text { and fracture }\end{array}$ & $\begin{array}{l}\text { Liu } \\
\text { Dongyang, } \\
\text { Guo Zhiyong } \\
\text { et al. } 33\end{array}$ \\
\hline $\begin{array}{l}\text { Bauhinia } \\
\text { brachycarpa Wall. ex } \\
\text { Benth. }\end{array}$ & $\begin{array}{l}\text { Miao: da fei } \\
\text { yang鞍叶羊 } \\
\text { 蹄甲an ye } \\
\text { yang ti jia }\end{array}$ & $\begin{array}{l}\text { Roots, } \\
\text { leaves }\end{array}$ & $\begin{array}{l}\text { Forest } \\
\text { edges }\end{array}$ & & $\begin{array}{l}\text { Roots treat diarrhea, } \\
\text { young leaves relieve } \\
\text { pain of fungal } \\
\text { diseases. Harvest } \\
\text { time: summer, } \\
\text { autumn }\end{array}$ & $\begin{array}{l}\text { Liu } \\
\text { Dongyang, } \\
\text { Guo Zhiyong } \\
\text { et al. } 16\end{array}$ \\
\hline $\begin{array}{l}\text { Caesalpinia } \\
\text { decapetala (Roth) } \\
\text { Alston. }\end{array}$ & $\begin{array}{l}\text { Miao: lao hu } \\
\text { ci jian } \\
\text { 云实yun shi }\end{array}$ & $\begin{array}{l}\text { Roots, } \\
\text { stems, } \\
\text { fruits }\end{array}$ & Thickets & $\begin{array}{l}\text { The bark } \\
\text { contains } \\
\text { tannins }\end{array}$ & $\begin{array}{l}\text { Roots treat cold and } \\
\text { rheumatic pain, } \\
\text { seeds are toxic. } \\
\text { Harvest time: } \\
\text { autumn }\end{array}$ & $\begin{array}{l}\text { Liu } \\
\text { Dongyang, } \\
\text { Guo Zhiyong } \\
\text { et al. } 17\end{array}$ \\
\hline
\end{tabular}




\begin{tabular}{|c|c|c|c|c|c|c|}
\hline $\begin{array}{l}\text { Senna tora (L.) } \\
\text { Roxb. }\end{array}$ & $\begin{array}{l}\text { Miao: ma ti } \\
\text { jue ming } \\
\text { 决明jue } \\
\text { ming }\end{array}$ & $\begin{array}{l}\text { Whole } \\
\text { plant }\end{array}$ & $\begin{array}{l}\text { Thickets } \\
\text { on slopes }\end{array}$ & $\begin{array}{l}\text { Seeds contain } \\
\text { anthracene } \\
\text { glycosides } \\
\text { substances } \\
\text { and xanthones } \\
\text { (Murshid et al. } \\
\text { 2007) }\end{array}$ & $\begin{array}{l}\text { Treat hypertension, } \\
\text { headaches and } \\
\text { constipation. } \\
\text { Harvest time: } \\
\text { autumn }\end{array}$ & $\begin{array}{l}\text { Liu } \\
\text { Dongyang, } \\
\text { Guo Zhiyong } \\
\text { et al. } 18\end{array}$ \\
\hline $\begin{array}{l}\text { Sophora flavescens } \\
\text { Alt. }\end{array}$ & $\begin{array}{l}\text { Miao: ye } \\
\text { huai } \\
\text { 苦参ku } \\
\text { shen }\end{array}$ & Roots & $\begin{array}{l}\text { Sandy } \\
\text { slopes }\end{array}$ & $\begin{array}{l}\text { Matrine, } \\
\text { cytisine et } \\
\text { al.(Lai et al. } \\
\text { 2003) }\end{array}$ & $\begin{array}{l}\text { Diuresis, antisepsis } \\
\text { and treat indigestion }\end{array}$ & $\begin{array}{l}\text { Liu } \\
\text { Dongyang, } \\
\text { Guo Zhiyong } \\
\text { et al. } 37\end{array}$ \\
\hline \multicolumn{7}{|l|}{ Lamiaceae } \\
\hline $\begin{array}{l}\text { Agastache rugosa } \\
\text { (Fisch. et Mey.) O. } \\
\text { Ktze. }\end{array}$ & $\begin{array}{l}\text { Miao: tu } \\
\text { huo xiang } \\
\text { 鹪香huo } \\
\text { xiang }\end{array}$ & $\begin{array}{l}\text { Whole } \\
\text { plant }\end{array}$ & $\begin{array}{l}\text { On acidic } \\
\text { soil, moist, } \\
\text { hot climate }\end{array}$ & Volatile oils & $\begin{array}{l}\text { Antiemetic, treat } \\
\text { abdominal pain. } \\
\text { Harvest time: } \\
\text { summer }\end{array}$ & $\begin{array}{l}\text { Liu } \\
\text { Dongyang, } \\
\text { Guo Zhiyong } \\
\text { et al. } 20\end{array}$ \\
\hline $\begin{array}{l}\text { Ajuga pantantha } \\
\text { Hand.-Mazz. }\end{array}$ & $\begin{array}{l}\text { Miao: dan } \\
\text { cao散瘀草 } \\
\text { san yu cao }\end{array}$ & $\begin{array}{l}\text { Whole } \\
\text { plant }\end{array}$ & Dry slope & & Anti-inflammatory & $\begin{array}{l}\text { Liu } \\
\text { Dongyang, } \\
\text { Guo Zhiyong } \\
\text { et al. } 21\end{array}$ \\
\hline \multicolumn{7}{|l|}{ Lardizabalaceae } \\
\hline $\begin{array}{l}\text { Sargentodoxa } \\
\text { cuneata (Oliv. ) } \\
\text { Rehd. et Wils. }\end{array}$ & $\begin{array}{l}\text { Miao: da } \\
\text { huo xue大 } \\
\text { 血藤da xue } \\
\text { teng }\end{array}$ & $\begin{array}{l}\text { Roots, } \\
\text { stems }\end{array}$ & $\begin{array}{l}\text { Hillside } \\
\text { thickets, } \\
\text { forest } \\
\text { edges }\end{array}$ & $\begin{array}{l}\text { Lignans (Han } \\
\text { et al. 1986) }\end{array}$ & $\begin{array}{l}\text { Activate collaterals, } \\
\text { trichomonacida }\end{array}$ & $\begin{array}{l}\text { Liu } \\
\text { Dongyang, } \\
\text { Guo Zhiyong } \\
\text { et al. } 10\end{array}$ \\
\hline \multicolumn{7}{|l|}{ Liliaceae } \\
\hline Paris polyphylla Sm. & $\begin{array}{l}\text { Miao: chong } \\
\text { lou七叶一枝 } \\
\text { 花qi ye yi } \\
\text { zhi hua }\end{array}$ & $\begin{array}{l}\text { Roots, } \\
\text { stems }\end{array}$ & $\begin{array}{l}\text { River } \\
\text { banks, } \\
\text { forests, } \\
2000-3000 \\
\mathrm{~m}\end{array}$ & $\begin{array}{l}\text { Polyphyllin } \\
\text { (Nohara et al. } \\
\text { 1973) }\end{array}$ & $\begin{array}{l}\text { Treat appendicitis. } \\
\text { Harvest time: } \\
\text { throughout the year }\end{array}$ & $\begin{array}{l}\text { Liu } \\
\text { Dongyang, } \\
\text { Guo Zhiyong } \\
\text { et al. } 23\end{array}$ \\
\hline \multicolumn{7}{|l|}{ Magnoliaceae } \\
\hline $\begin{array}{l}\text { Illicium verum Hook. } \\
\text { f. }\end{array}$ & $\begin{array}{l}\text { Miao: ba } \\
\text { jiao hui } \\
\text { xiang } \\
\text { 八角ba jiao }\end{array}$ & $\begin{array}{l}\text { Fruits, } \\
\text { leaves }\end{array}$ & $\begin{array}{l}\text { Mountain } \\
\text { climate }\end{array}$ & $\begin{array}{l}\text { Anisylacetone, } \\
\text { fatty oil, } \\
\text { protein, resin, } \\
\text { shikimic } \\
\text { acid.(Zheng- } \\
\text { Cai et al. } \\
\text { 2013) }\end{array}$ & $\begin{array}{l}\text { Regulate breath, } \\
\text { treat neurasthenia. } \\
\text { Harvest time: spring, } \\
\text { autumn }\end{array}$ & $\begin{array}{l}\text { Liu } \\
\text { Dongyang, } \\
\text { Guo Zhiyong } \\
\text { et al. } 24\end{array}$ \\
\hline \multicolumn{7}{|l|}{ Oleaceae } \\
\hline $\begin{array}{l}\text { Fraxinus chinensis } \\
\text { Roxb. }\end{array}$ & $\begin{array}{l}\text { Miao: nv } \\
\text { zhen } \\
\text { 白蜡树bai la } \\
\text { shu }\end{array}$ & $\begin{array}{l}\text { Whole } \\
\text { plant }\end{array}$ & $\begin{array}{l}\text { Mixed } \\
\text { forests }\end{array}$ & & $\begin{array}{l}\text { Seeds nourishing } \\
\text { liver and kidney, } \\
\text { leaves treat } \\
\text { stomatitis, bark } \\
\text { treats burns, roots } \\
\text { treat rheumatism. } \\
\text { Harvest time: } \\
\text { summer, autumn }\end{array}$ & $\begin{array}{l}\text { Liu } \\
\text { Dongyang, } \\
\text { Guo Zhiyong } \\
\text { et al. } 25\end{array}$ \\
\hline $\begin{array}{l}\text { Fraxinus } \\
\text { malacophylla Hemsl. }\end{array}$ & $\begin{array}{l}\text { Miao: bai } \\
\text { qiang gan } \\
\text { 白枪杆bai } \\
\text { qiang gan }\end{array}$ & Roots & Forests & $\begin{array}{l}\text { Tannins (Guo } \\
\text { et al. 2012) }\end{array}$ & $\begin{array}{l}\text { Diminish } \\
\text { inflammation, } \\
\text { diuretic, aperient, } \\
\text { digestion and } \\
\text { stomachic. Harvest } \\
\text { time: autumn, winter }\end{array}$ & $\begin{array}{l}\text { Liu } \\
\text { Dongyang, } \\
\text { Guo Zhiyong } \\
\text { et al. } 6\end{array}$ \\
\hline $\begin{array}{l}\text { Jasminum sambac } \\
\text { (L.) Aiton }\end{array}$ & 茉莉mo li & $\begin{array}{l}\text { Flowers, } \\
\text { roots }\end{array}$ & Thickets & $\begin{array}{l}\text { Benzyl alcohol } \\
\text { and ester } \\
\text { (Gmm et al. } \\
2014)\end{array}$ & $\begin{array}{l}\text { Flowers treat fever } \\
\text { and are used to } \\
\text { detoxification. Roots } \\
\text { are toxic, which } \\
\text { have analgesic } \\
\text { effect. Harvest time: } \\
\text { autumn }\end{array}$ & $\begin{array}{l}\text { Liu } \\
\text { Dongyang, } \\
\text { Guo Zhiyong } \\
\text { et al. } 27\end{array}$ \\
\hline \multicolumn{7}{|l|}{ Orchidaceae } \\
\hline $\begin{array}{l}\text { Bletilla striata } \\
\text { (Thunb. ex A. } \\
\text { Murray) Rchb. f. }\end{array}$ & $\begin{array}{l}\text { Miao: bai } \\
\text { gen } \\
\text { 白及bai ji }\end{array}$ & $\begin{array}{l}\text { Dry } \\
\text { tubers }\end{array}$ & $\begin{array}{l}\text { Broad- } \\
\text { leaved or } \\
\text { coniferous } \\
\text { forest }\end{array}$ & $\begin{array}{l}\text { Militarine } \\
\text { (Sakuno et al. } \\
\text { 2010) }\end{array}$ & $\begin{array}{l}\text { Treat } \\
\text { gastrointestinal } \\
\text { bleeding, traumatic } \\
\text { bleeding. Harvest } \\
\text { time: autumn }\end{array}$ & $\begin{array}{l}\text { Liu } \\
\text { Dongyang, } \\
\text { Guo Zhiyong } \\
\text { et al. } 28\end{array}$ \\
\hline
\end{tabular}




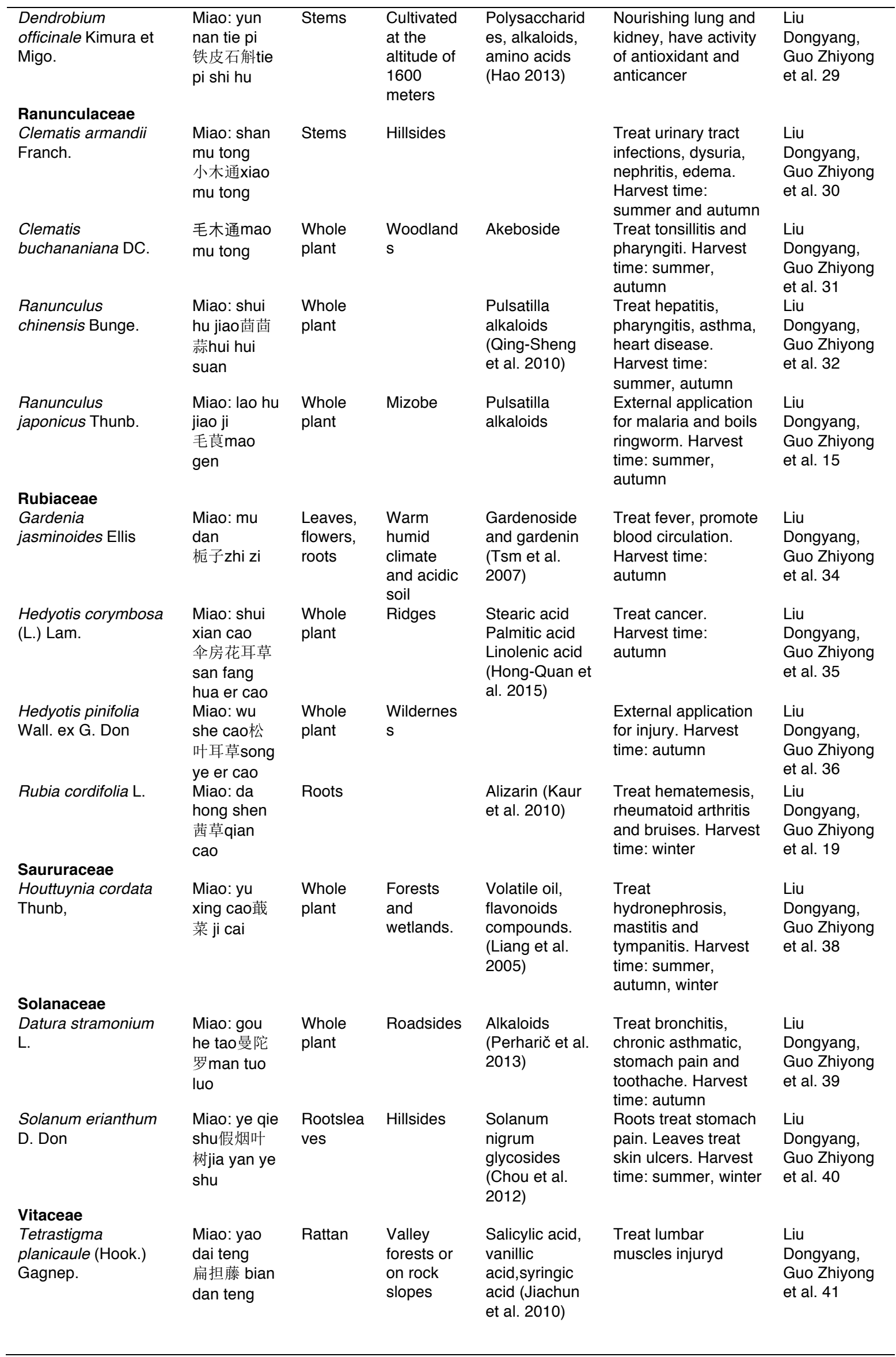




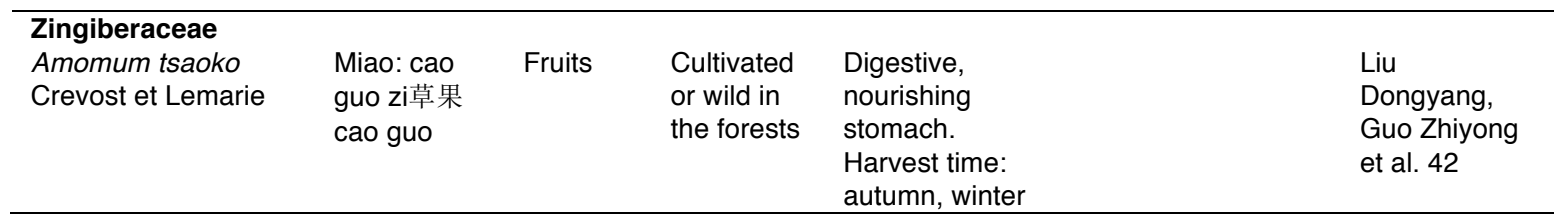

Common diseases and common Miao botanical prescriptions in Jijiezi

An overview on the most important illness categories treated and the plants used is given in Table 3. Commonly treated diseases are closely related to local environmental conditions, lifestyle and dietary habit, and herbs for curing common diseases such as bruises, rheumatic disorders, cold and diarrhea, were used with a higher frequency. Meanwhile Miao medicine is mainly used for the treatment of snake bites, rheumatism, kidney stones, wounds and other tropical diseases.

Table 3. Common diseases treated by Miao medicines

\begin{tabular}{|c|c|}
\hline Common & Main species used \\
\hline $\begin{array}{l}\text { Activating blood } \\
\text { circulation, dissipate } \\
\text { bruises }\end{array}$ & $\begin{array}{l}\text { Bauhinia brachycarpa Wall. ex Benth., Rubia cordifolia L., Hedyotis pinifolia } \\
\text { Wall. ex G. Don, Solanum verbascifolium L., Alysicarpus vaginalis (L.) DC., } \\
\text { Antenoron filiforme (Thunb.) Rob. et Vaut., Chloranthus japonicus Sieb., } \\
\text { Sargentodoxa cuneata (Oliv.) Rehd. et Wils., Paris polyphylla Sm., Arisaema } \\
\text { heterophyllum Blume, Tetrastigma planicaule (Hook.) Gagnep., Gardenia } \\
\text { jasminoides Ellis. }\end{array}$ \\
\hline Anthelmintic & Sargentodoxa cuneata (Oliv. ) Rehd. et Wils., Sophora flavescens Alt. \\
\hline Antidiarrheal & $\begin{array}{l}\text { Caesalpinia decapetala (Roth) Alston, Bauhinia brachycarpa Wall. ex Benth., } \\
\text { Allium sativum L., Ligusticum sinense Oliv., Carica papaya L. }\end{array}$ \\
\hline Antiphlogistic & $\begin{array}{l}\text { Phyllanthus emblica L., Fraxinus malacophylla Hemsl., Fraxinus chinensis } \\
\text { Roxb., Ranunculus chinensis Bunge, Clematis buchananiana DC., Datura } \\
\text { stramonium L., Ajuga pantantha Hand.-Mazz., Paris polyphylla Sm., Bletilla } \\
\text { striata (Thunb. ex A. Murray) Rchb. f., Ligusticum sinense Oliv., Platycodon } \\
\text { grandiflorus (Jacq.) A. DC., Sophora flavescens Alt. }\end{array}$ \\
\hline Burns and scalds & Fraxinus chinensis Roxb., Bletilla striata (Thunb. ex A. Murray) Rchb. f. \\
\hline Cold & $\begin{array}{l}\text { Phyllanthus emblica L., Caesalpinia decapetala (Roth) Alston, Allium sativum L., } \\
\text { Ranunculus chinensis Bunge, Ranunculus japonicus Thunb., Chloranthus } \\
\text { japonicus Sieb., Paris polyphylla Sm., Ligusticum sinense Oliv., Platycodon } \\
\text { grandiflorus (Jacq.) A. DC., Capparis masaikai Levl. }\end{array}$ \\
\hline $\begin{array}{l}\text { Fever, } \\
\text { Detoxification }\end{array}$ & $\begin{array}{l}\text { Jasminum sambac (L.) Aiton, Ajuga pantantha Hand.-Mazz., Arisaema } \\
\text { heterophyllum Blume, Sophora flavescens Alt., Capparis masaikai Levl., } \\
\text { Gardenia jasminoides Ellis, Paris polyphylla Sm., Chloranthus japonicus Sieb. }\end{array}$ \\
\hline $\begin{array}{l}\text { Intestines and } \\
\text { stomach disease }\end{array}$ & $\begin{array}{l}\text { Rubia cordifolia L., Phyllanthus emblica L., Fraxinus malacophylla Hemsl., } \\
\text { Datura stramonium L., Agastache rugosa (Fisch. et Mey.) O. Ktze., Antenoron } \\
\text { filiforme (Thunb.) Rob. et Vaut., Amomum tsaoko Crevost et Lemarie, Illicium } \\
\text { verum Hook. f., Dendrobium officinale Kimura et Migo, Bletilla striata (Thunb. ex } \\
\text { A. Murray) Rchb. f., Houttuynia cordata Thunb, Carica papaya L., Solanum } \\
\text { verbascifolium L. }\end{array}$ \\
\hline $\begin{array}{l}\text { Lowering blood } \\
\text { pressure }\end{array}$ & $\begin{array}{l}\text { Phyllanthus emblica L., Senna tora (L.) Roxb., Dendrobium officinale Kimura et } \\
\text { Migo, Platycodon grandiflorus (Jacq.) A. DC., Eucommia ulmoides Oliver I. c. }\end{array}$ \\
\hline Nephritis, calculi & $\begin{array}{l}\text { Clematis armandii Franch., Clematis buchananiana DC., Dendrobium officinale } \\
\text { Kimura et Migo, Houttuynia cordata Thunb, Eucommia ulmoides Oliver }\end{array}$ \\
\hline $\begin{array}{l}\text { Pulmonary } \\
\text { tuberculosis }\end{array}$ & Phyllanthus emblica L., Allium sativum L. \\
\hline Rheumatism & $\begin{array}{l}\text { Rubia cordifolia L., Lycoris aurea (L'Hér.) Herb., Caesalpinia decapetala (Roth) } \\
\text { Alston, Bauhinia brachycarpa Wall. ex Benth., Fraxinus chinensis Roxb., } \\
\text { Clematis armandii Franch., Chloranthus japonicus Siebold, Sargentodoxa } \\
\text { cuneata (Oliv. ) Rehd. et Wils., Tetrastigma planicaule (Hook.) Gagnep. }\end{array}$ \\
\hline Snakebite & $\begin{array}{l}\text { Alysicarpus vaginalis (L.) DC., Chloranthus japonicus Sieb., Paris polyphylla } \\
\text { Sm., Arisaema heterophyllum Blume. }\end{array}$ \\
\hline
\end{tabular}


Miao medicine is divided into cold medicine and hot medicine. It follows the principle "cold disease with hot drugs, fever with cold medicine". Sweet herbs are regarded as hot, bitter and astringent plants as cold. Miao medicine normally prefers fresh herbs. The local people plant commonly used medicinal plants around their houses.

\section{The status quo of local Miao knowledge} Prospect of Miao medicine
With increasing demand in the domestic and international medicine markets, the Miao people have started to establish small production factories for medicinal plants commonly used in traditional medicine (Fig. 4), such as Dendrobium officinale Kimura et Migo, Eucommia ulmoides Oliver, Gardenia jasminoides Ellis, Carica papaya L., Paris polyphylla Sm., Antenoron filiforme (Thunb.) Rob. et Vaut.
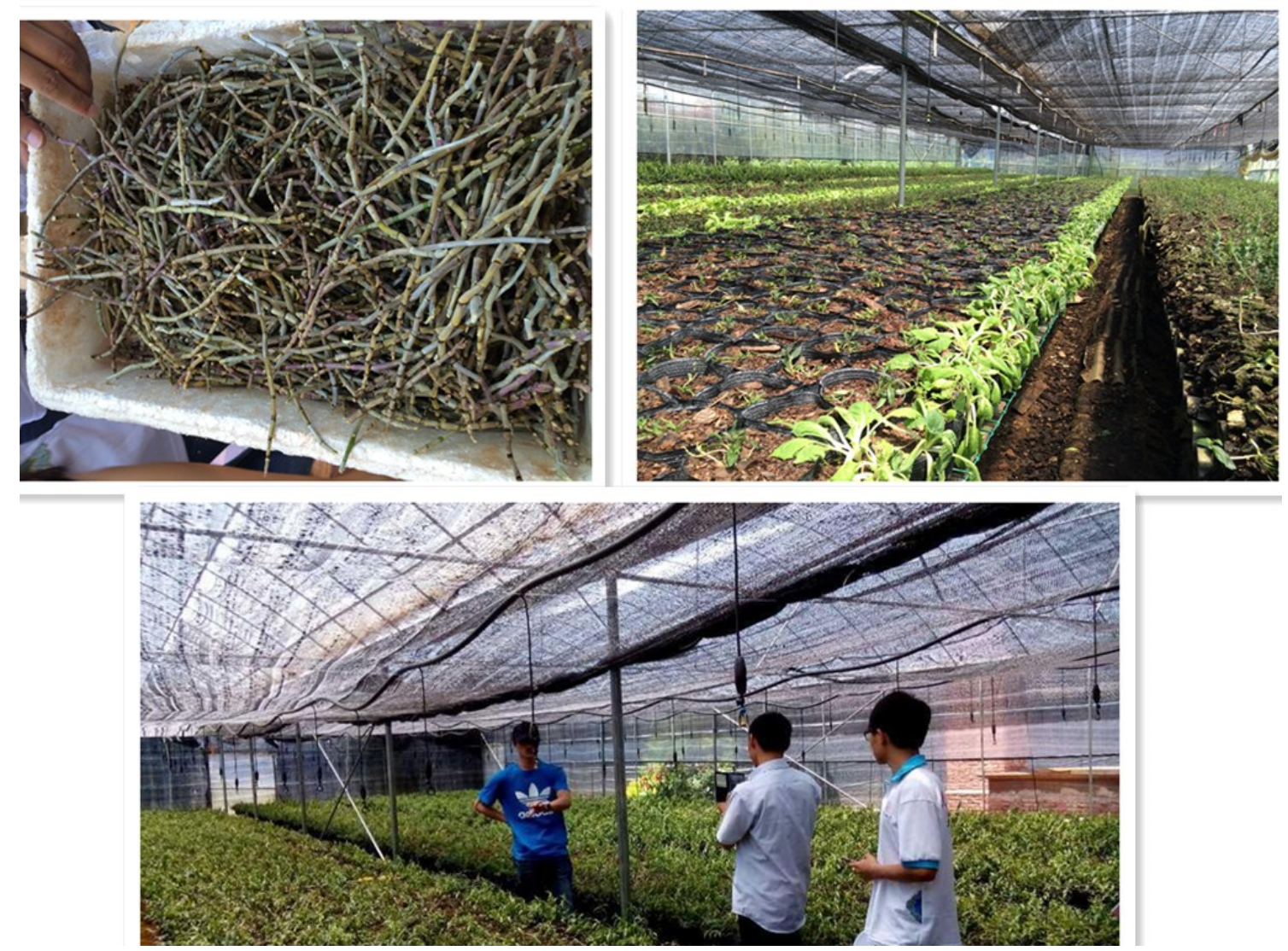

Fig. 4. Dendrobium officinale Kimura et Migo

Our study found that many medicinal herbs were still abundant, including Sargentodoxa cuneata (Oliv. ) Rehd. et Wils., Antenoron filiforme (Thunb.) Rob. et Vaut., Chloranthus japonicus Siebold, Amomum tsaoko Crevost et Lemarie, Illicium verum Hook. f., Lonicera japonica Thunb., Paris polyphylla Sm., Dendrobium officinale Kimura et Migo, Bletilla striata (Thunb. ex A. Murray) Rchb. f. and Ligusticum sinense Oliv.

Local people ate Phyllanthus emblica L. every day to reduce "pathogenic fire". Capparis masaikai Levl. is also used as a sweetener (Hu \& He 1983).

Potential crisis of local Miao medicine

The development of the Miao is lagging behind the Han because of historical reasons. In recent history, the Miao were included in the modern society, and traditional culture was influenced and interrupted by external forces.

The statistics of Miao medicine use by local farmers is shown in Fig. 5. In order to understand the statistics of cognizing Miao medicine by Miao people, we used the simple formula:

$$
\mathrm{F}=\mathrm{N}_{\mathrm{K}} / \mathrm{N}_{\mathrm{S}}
$$

Where, $\mathrm{N}_{k}$ is the sum of people who know the number of types of Miao medicine in the interview, $\mathrm{Ns}$ is the total number of people who were interviewed. $F$ is the proportion of cognizing the number of different types of Miao medicines in the total number of people. We can use "F" to describe the status of the Miao medicine because we can 
know the status of local people's cognizing of Miao medicine from "F".

The participants showed a widely varying knowledge of medicinal plants. Most people knew 5 to 19 species, with $46 \%$ of the interviewees knowing less than 9 species. About $20 \%$ of the participants knew more than 20 species, $50 \%$ people recognized $10-29$ species of medicinal herbs and a few $(4 \%)$ recognize more than 30 medicinal herbs. In this study, we also noticed that almost all adolescents kew fewer than 10 (Fig. 5). Medicinal knowledge was mainly kept within individual families.
The frequency of using Miao medicine by Miao people depends on gender and age. In general, women use Miao medicine more frequently than men in the same age group, and older people with higher frequency than younger people (Fig. 6). The highest frequency group of people using traditional medicine were is the elderly women, followed by older men $(28.4 \%)$, adult women (19.75\%), adult men $(13.58 \%)$, and adolescents with the lowest usage rate $(6.17 \%)$, indicating a loss of interest in the knowledge of Miao medicine.

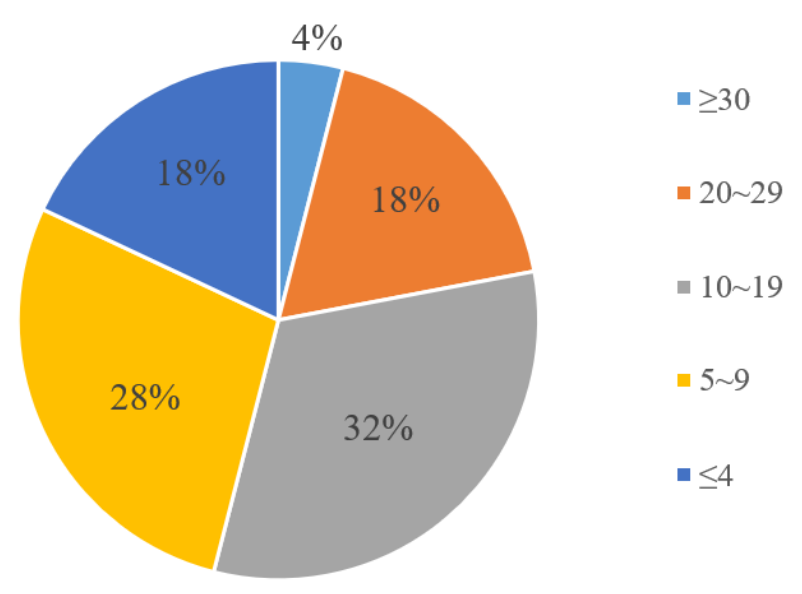

Fig. 5. Number of Miao medicinal plant species recognized by the 200 participants

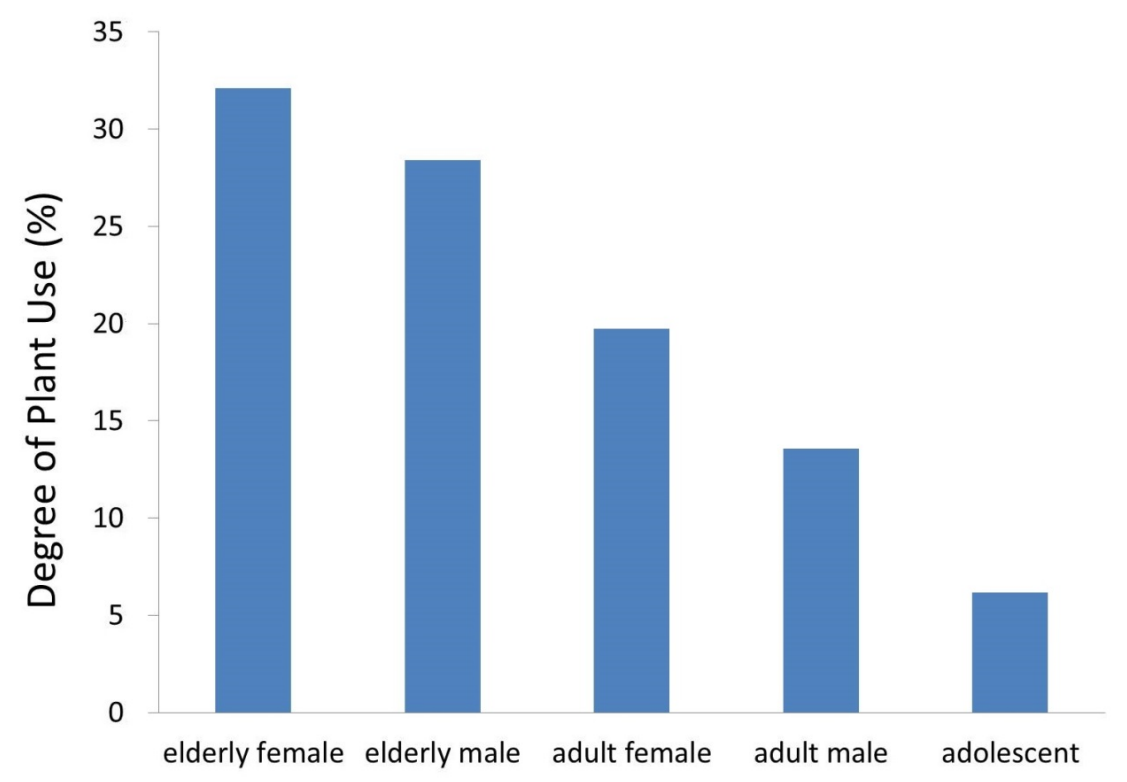

Fig. 6. Demographics of local people who used Miao medicines 
Due to the strong impact of modern western medicine, as well as Traditional Chinese Medicine, many young people have lost interest in Miao medicine, and very few traditional Miao practitioners have apprentices at the moment. In addition, practitioners of Miao medicine normally have a very low income, and there is little economic incentive for the younger generation to learn the tradition, because other income sources are much more lucrative. The practitioners of Miao medicine are very traditional, conservative, and honor the value of the knowledge from their ancestors. Therefore, they do prefer to rather let the knowledge be lost, than to spread ancestral knowledge to strangers. The education level of most practitioners is low, and many practitioners of Miao medicine do not speak Chinese, which makes the documentation and translation of Miao medicine more difficult.

\section{Conclusions}

Based on the investigation we give the following suggestions to provide the basis for the development of Miao medicine.

(1) It is necessary to strengthen the documentation of Miao medicine prescriptions, diagnoses and treatment technology and efficacy. The knowledge of Miao medicine is decliningdue to lack of apprentices.

(2) Establish an apprentice program for Miao medicine, because with the death of many Miao practitioners, as well as the influence of modern medicine, the knowledge of Miao medicine is being lost.

(3) Through policy support, strengthening the search and protection of intangible cultural heritage.

(4) Local government should bring Miao medicine to the market to promote the inheritance and continuation of Miao medicine cultural through economic mode.

\section{Declarations}

Ethics approval and consent to participate: The semi-structured interviews were carried out anonymous, and all the participants involved in this study gave their informed consent. Permissions were provided by all participants in this study. Consent was obtained from the participants prior to this study being carried out. The authors have all copyrights. Institutional review board approval of Minzu University of China was obtained for this study.

Consent for publication: Not applicable

Availability of data and materials: We are willing to share data generated or analyzed during the current study.
Competing interests: The authors declare that they have no competing interests.

Funding: Strategic Priority Research Program of the Chinese Academy of Sciences (Grant No. XDA19050404), Survey and Germplasm Conservation of Plant Species with Extremely Small Populations in Southwest China (2017FY100100), and National Natural Science Foundation of China (31400182), the foundation of Key Laboratory of Ethnomedicine (Minzu University of China) (No.KLEM-ZZ201905), Global Environment Facility Small Grants Programme (UNDP).

\section{Authors' contributions}

Conceived of the study: BL; Conducted surveys: DL, HC, ZG; Identified plant species: BL, HJH; Analyzed data: DL and HC; Wrote the manuscript: DL, HC, RB, ZG, BL; Edited the manuscript: BL, RB, DL, HC, ZG. All authors read and approved the final manuscript.

\section{Acknowledgements}

We thank the villagers who accepted to be interviewed and helped us in our work. We also thank Yi LIU and Yi DENG for the training of endogenous community sustainable development workshop.

\section{Literature cited}

Anderson DM, Salick J, Moseley RK, Xiaokun O. 2005. Conserving the Sacred Medicine Mountains: A Vegetation Analysis of Tibetan Sacred Sites in Northwest Yunnan. Biodiversity and Conservation. 14(13): 3065-3091.

Baghalian K, Ziai SA, Naghavi MR, Badi HN, Khalighi A. 2005. Evaluation of allicin content and botanical traits in Iranian garlic (Allium sativum L.) ecotypes. Scientia Horticulturae. 103(2): 155-166.

Baiming AT, Ren FX, Yang Y, Zhang Y, Qu AT., Zhao YM. 2012. The chemical constituents of Bauhinia glauca (Wall, ex Benth.) Benth. subsp. hupehana (Craib) T. Chen. Journal of Chinese Pharmaceutical Sciences. 47(22): 1796-1798.

Blania G, Spangenberg B. 1991. Formation of allicin from dried garlic (Allium sativum): a simple HPTLC method for simultaneous determination of allicin and ajoene in dried garlic and garlic preparations. Planta Medica. 57(4): 371-375.

Canini A, Alesiani D, D'Arcangelo G, Tagliatesta P. 2007. Gas chromatography-mass spectrometry analysis of phenolic compounds from Carica papaya L. leaf. Journal of Food Composition \& Analysis. 20(7): 584-590. 
Chen SH, Su JY, Wu MJ. 2010. Hedyotis pinifolia Wall. ex G. Don (Rubiaceae), a New Record to the Flora of Taiwan. Taiwania. 55.

Chen Y, Liu J, Davidson RS, Howarth OW. 1993. Isolation and Structure of Clematine (I), a New Flavanone Glycoside from Clematis armandii Franch. Tetrahedron. 49(23):5169-5176.

Cheng L, Meng XB, Lu S, Wang TT, Liu Y, Sun GB, Sun XB. 2013. Evaluation of hypoglycemic efficacy of tangningtongluo formula, a traditional Chinese Miao medicine, in two rodent animal models. Journal of Diabetes Research. 2014(2014): 745419-745419. Chou SC, Huang TJ, Lin EH, Huang $\mathrm{CH}$, Chou $\mathrm{CH}$. 2012. Antihepatitis B virus constituents of Solanum erianthum. Natural Product Communications. 7(2): 153-156.

Gmm M, Ak B, Mm R. 2014. Evaluation of antioxidant, analgesic and cytotoxic activities of the aerial part of Cassia sophera L. (Caesalpiniaceae). Phytomedicine International Journal of Phytotherapy \& Phytopharmacology. 5(2014): 383-389.

Guo LJ, Tan XQ, Zheng W. 2012. Chemical constituents of Fraxinus malacophylla Hemsl.[II]. Central South Pharmacy. 10(9): 661-663.

Han GQ, Chang MN, Hwang SB. 1986. [The investigation of lignans from Sargentodoxa cuneata (Oliv) Rehd et Wils. Yao xue xue bao. Acta pharmaceutica Sinica. 21(1): 68-70.

Hao LI. 2013. Advance in Studies on Chemical Constituents in Dendrobium officinale. Chinese Journal of Spectroscopy Laboratory.

Hong-Quan LI, Bai YB, Zhou YM, Xie JC, Xia BH, Lin LM. 2015. Content determination of ursolic acid and oleanolic acid in Hedyotis cormbosa (L.) Lam. China Modern Medicine. 22(8):12-15.

Hu G, Sheng C, Mao R, Ma Z, Lu Y, Wei D. 2013. Essential oil composition of Allium tuberosum seed from China. Chemistry of Natural Compounds. 48(6): 1091-1093.

Hu S, Hu S, Hu Q. 2003. Medicament for treating aseptic inflammations containing anemonin as effective component. US.

Hu Z, He M. 1983. Studies on Mabinlin, a sweet protein from the seeds of Capparis masaikai Levl. I. Extraction, purification and certain characteristics. Yun Nan Chi Wu Yen Chiu Acta Botanica Yunnanica. 5: 207-212.

Jäger AK, Gauguin B, Adsersen A, Gudiksen L. 2006. Screening of plants used in Danish folk medicine to treat epilepsy and convulsions. Journal of Ethnopharmacology. 105(1-2): 294-300.

Janes CR. 1999. The Health Transition, Global Modernity and the Crisis of Traditional Medicine: The
Tibetan Case. Social Science \& Medicine. 48(12): 1803-1820.

Jiachun S, Cuihong $\mathrm{H}$, Ting L, Aihua $\mathrm{H}$, Yingzhou C. 2010. Studies on the chemical constituents of Tetrastigma planicaule (Hook.) Gagnep. Chinese Pharmaceutical Journal. 45(21): 1615-1617.

Kaur P, Chandel M, Kumar S, Kumar N, Singh B, Kaur S. 2010. Modulatory role of alizarin from Rubia cordifolia L. against genotoxicity of mutagens. Food \& Chemical Toxicology. 48(1): 320-325.

Lai JP, He XW, Jiang Y, Chen F. 2003. Preparative separation and determination of matrine from the Chinese medicinal plant Sophora flavescens Ait. by molecularly imprinted solid-phase extraction. Analytical \& Bioanalytical Chemistry. 375(2): 264269.

Liang M, Qi M, Zhang C, Zhou S, Fu R, Huang J. 2005. Gas chromatography-mass spectrometry analysis of volatile compounds from Houttuynia cordata Thunb. after extraction by solid-phase microextraction, flash evaporation and steam distillation. Analytica Chimica Acta. 531(1): 97-104. Long CL, Rong L. 2004. Ethnobotanical studies on medicinal plants used by the Red-headed Yao People in Jinping, Yunnan Province, China. Journal of Ethnopharmacology. 90(2-3): 389-395.

Lovett JV, Potts WC. 1987. Primary effects of allelochemicals of Datura stramonium L. Plant \& Soil. 98(98): 137-144.

Murshid GMM, Moniruzzaman M, Rahman AA, Saifuzzaman M, Uddin SN. 2007. Phytochemical and Pharmacological Screening of Senna tora Roxb. Journal of Pharmacology \& Toxicology. 2(4): 386390.

Nohara T, Yabuta H, Suenobu M, Hida R, Miyahara K, Kawasaki T. 1973. Steroid Glycosides in Paris polyphylla SM. Chemical \& Pharmaceutical Bulletin. 21(6): 1240-1247.

Pan DZ. 1996. On the cultural spirit of chiyou and Miao nationality from the inheritance culture of Miao people. Journal of Guizhou University for Ethnic Mino rities (Philosophy and social science) (4).

Perharič L, Koželj G, Družina B, Stanovnik L. 2013. Risk assessment of buckwheat flour contaminated by thorn-apple (Datura stramonium L.) alkaloids: a case study from Slovenia. Food Additives \& Contaminants Part A. 30(2): 321-330.

Li QS, Li J, Yin HL, Dong JX. 2010. Chemical constituents of Rannunculus chinensis Bunge. Bulletin of the Academy of Military Medical Sciences. 34(1): 68-70.

Racines AGM. 2006. Indigenous and underutilized Senna tora (L.) Roxb. is a potential species for food, 
feed, medicine and climate change adaptation. Electroanalysis. 18(9): 862-872.

Sakuno E, Kamo T, Takemura T, Sugie, H., Hiradate, S., Fujii, Y. 2010. Contribution of militarine and dactylorhin $A$ to the plant growth-inhibitory activity of a weed-suppressing orchid, Bletilla striata. Weed Biology \& Management.10(3): 202-207.

Shen S, Qian J, Ren J. 2010. Ethnoveterinary plant remedies used by $\mathrm{Nu}$ people in NW Yunnan of China. Journal of Ethnobiology and Ethnomedicine. 6(1): 24.

Tong J, Wang R, Li X, Wang Y, Peng F, Bing X. 2011. Gene cloning and amino acid sequence analysis of ribosomal protein L21 from Lycoris radiata. Journal of Plant Resources and Environment. 20(4): 13-16.

Tsai PS, Lee PH, Wang MY. 2008. Demographics, training, and practice patterns of practitioners of folk medicine in Taiwan: a survey of the Taipei metropolitan area. Journal of Alternative \& Complementary Medicine. 14(10): 1243-1248.

Tsm TH, Westly J, Lee TF, Chen CF. 2007. Identification and Determination of Geniposide, Genipin, Gardenoside, and Geniposidic Acid from Herbs by HPLC/Photodiode-Array Detection. Journal of Liquid Chromatography. 17(10): 21992205.

Ueda M, Tashiro C, Yamamura S. 1997. cis-pCoumaroylagmatine, the genuine leaf-opening substance of a nyctinastic plant, Albizia julibrissin Durazz. Tetrahedron Letters. 38: 3253-3256.

Wei XH, Yang SJ, Liang N, Hu DY, Jin LH, Xue W, Yang S. 2013. Chemical constituents of Caesalpinia decapetala (Roth) Alston. Molecules. 18(1): 13251336.

Wei XL, Yang CH. 2005. Constituents of the barks of Fraxinus chinensis Roxb. Chinese Journal of Natural Medicines. 3(4): 228-230.

Wu L, Zhang P. 2005. Study on Flavor Compositions of Allium tuberosum Rottler by GCM/ S. Guangzhou Chemical Industry. 32(7): 67-68.

Wu XP. 2004. Shamenism among the Hmong People of the USA. Journal o f Guizhou University for Ethnic Mino rities (Philosophy and social science) (1).

Xiong Y, Cheng ZG, Peng ZY, Wang ZL. 2010. Miao medicine Shengxian soup and herbal fumigation unite treatment knee osteoarthritis 120 cases clinical curative effects observation. Liaoning Journal of Traditional Chinese Medicine. 37(12): 2397-2399.

Xu TH, Li HT, Xu YJ, Zhao HF, Xie SX, Han D, Si YS, Li Y, Niu JZ, Xu DM. 2008. A new triterpenoid saponin from Albizia julibrissin Durazz. Journal of Asian Natural Products Research. 10(7-8): 781-785.
Ju Y, Zhuo JX, Liu B, Long CL. 2012. Eating from the wild: diversity of wild edible plants used by Tibetans in Shangri-la region, Yunnan, China. Journal of Ethnobiology \& Ethnomedicine. 9(1): 169-176.

Zhang Z, Zhang L, Li LQ. 2015. Artificial propagation analysis on wild edible fungus in Yimen county. Forestry Construction. 1: 33-35.

Du ZC, Li XJ, Huang YX, Lin Y, Zhou ZX, Deng JG, Hu, WJ. 2013. Influence of Distillation Methods on Extraction of Star Anise Oil and Shikimic Acid in Illicium verum Hook. f. Journal of Guangxi University of Chinese Medicine.

Zheng J. 2006. Reflection on Enhancing Works of Inheriting and Developing Nationalistic Medicine in Yunnan Province. Journal of Yunnan University of Traditional Chinese Medicine. 29(1): 3-6.

Zheng YZ, Zhang ZX, Dong TX, Wah-Keung TK, University HN. 2013. Analysis of HPLC Fingerprints and Determination of Gallic Acid and Ellagic Acid of Phyllanthi Fructus from Phyllanthus emblica. Chinese Journal of Experimental Traditional Medical Formulae. 23: 94-99. 